\title{
A U.S. isolate of Theileria orientalis, Ikeda genotype, is transmitted to cattle by the invasive Asian longhorned tick, Haemaphysalis longicornis
}

Kelcey D. Dinkel ${ }^{1 \dagger}$, David R. Herndon ${ }^{2 \dagger}$, Susan M. Noh ${ }^{1,2}$, Kevin K. Lahmers ${ }^{3}$, S. Michelle Todd ${ }^{3}$, Massaro W. Ueti ${ }^{1,2}$, Glen A. Scoles ${ }^{2,4}$, Kathleen L. Mason ${ }^{2}$ and Lindsay M. Fry ${ }^{1,2^{*}}$

\begin{abstract}
Background: Theileria orientalis is a tick-borne hemoparasite that causes anemia, ill thrift, and death in cattle globally. The Ikeda strain of T. orientalis is more virulent than other strains, leading to severe clinical signs and death of up to $5 \%$ of affected animals. Within the Asia-Pacific region, where it affects $25 \%$ of Australian cattle, T. orientalis Ikeda has a significant economic impact on the cattle industry. In 2017, T. orientalis Ikeda was detected in a cattle herd in Albermarle County, Virginia, United States. Months earlier, the U.S. was alerted to the invasion of the Asian longhorned tick, Haemaphysalis longicornis, throughout the eastern U.S. Abundant H. longicornis ticks were identified on cattle in the T. orientalis-affected herd in VA, and a subset of ticks from the environment were PCR-positive for T. orientalis Ikeda. A strain of T. orientalis from a previous U.S. outbreak was not transmissible by H. longicornis; however, H. longicornis is the primary tick vector of T. orientalis Ikeda in other regions of the world. Thus, the objective of this study was to determine whether invasive H. longicornis ticks in the U.S. are competent vectors of T. orientalis Ikeda.

Methods: Nymphal H. longicornis ticks were fed on a splenectomized calf infected with the VA-U.S.-T. orientalis Ikeda strain. After molting, a subset of adult ticks from this cohort were dissected, and salivary glands assayed for T. orientalis Ikeda via qPCR. The remaining adult ticks from the group were allowed to feed on three calves. Calves were subsequently monitored for T. orientalis Ikeda infection via blood smear cytology and PCR.

Results: After acquisition feeding on a VA-U.S.-T. orientalis Ikeda-infected calf as nymphs, a subset of molted adult tick salivary glands tested positive by qPCR for T. orientalis Ikeda. Adult ticks from the same cohort successfully transmitted T. orientalis Ikeda to $3 / 3$ naïve calves, each of which developed parasitemia reaching $0.4-0.9 \%$.

Conclusions: Our findings demonstrate that U.S. H. longicornis ticks are competent vectors of the VA-U.S.-T. orientalis Ikeda strain. This data provides important information for the U.S. cattle industry regarding the potential spread of this parasite and the necessity of enhanced surveillance and control measures.
\end{abstract}

Keywords: Theileria orientalis, Ikeda genotype, Haemaphysalis longicornis, Asian longhorned tick, Transmission, Cattle

\footnotetext{
*Correspondence: Lindsay.Fry@USDA.gov

${ }^{\dagger}$ KelceyD. Dinkel and DavidR. Herndon contributed equally to this work

${ }^{1}$ Department of Veterinary Microbiology \& Pathology, Washington State University, Pullman, WA, USA

Full list of author information is available at the end of the article
}

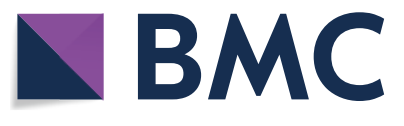

(c) The Author(s) 2021. This article is licensed under a Creative Commons Attribution 4.0 International License, which permits use, sharing, adaptation, distribution and reproduction in any medium or format, as long as you give appropriate credit to the original author(s) and the source, provide a link to the Creative Commons licence, and indicate if changes were made. The images or other third party material in this article are included in the article's Creative Commons licence, unless indicated otherwise in a credit line to the material. If material is not included in the article's Creative Commons licence and your intended use is not permitted by statutory regulation or exceeds the permitted use, you will need to obtain permission directly from the copyright holder. To view a copy of this licence, visit http://creativeco mmons.org/licenses/by/4.0/. The Creative Commons Public Domain Dedication waiver (http://creativecommons.org/publicdomain/ zero/1.0/) applies to the data made available in this article, unless otherwise stated in a credit line to the data. 


\section{Background}

Theileria orientalis comprises a diverse group of nontransforming, bovine hemoparasites that were formerly classified as T. sergenti and T. buffeli [1]. Phylogenetic analysis using major piroplasm surface protein (MPSP) sequences of numerous isolates identified 11 allelic genotypes [2], with the Buffeli, Chitose, p32, and Ikeda genotypes being most prevalent in Japan, Australia, New Zealand, and the U.S [2, 3]. T. orientalis genotypes vary widely in virulence [4]. The Ikeda genotype results in severe infection in $1-6 \%$ of infected animals $[5,6]$, while infection with the Buffeli genotype is almost always clinically silent $[3,5]$. Moderate to severe infections are characterized by erythrocyte destruction, leading to anemia and hypoxia. Severely affected animals often exhibit pyrexia, weakness, pallor, and increased heart and respiratory rates $[7,8]$. Abortion is a common sequela to T. orientalis Ikeda infection, although transplacental transmission of the parasite from cows to calves is only observed in $10 \%$ of cases $[9,10]$.

The primary tick vector of $T$. orientalis is Haemaphysalis longicornis (AKA the Asian longhorned tick) $[3,11]$; however, other tick species $[3,12]$ and mechanical vectors, including contaminated needles [13], biting flies [13], and lice [14], have been implicated in parasite spread. Regardless, $T$. orientalis Ikeda can spread rapidly and cause significant losses when introduced to naïve cattle in areas with competent tick vectors. T. orientalis Ikeda was first identified in Australia in 2011 [15], and by 2014, outbreaks of the disease had affected approximately $25 \%$ of Australian cattle $[16,17]$. It is estimated that the indirect costs of $T$. orientalis Ikeda to the Australian beef industry, comprised of reduced meat and milk yields $[16,18]$, is $\$ 19.6$ million per year [19]. A similar, sudden emergence of the $T$. orientalis Ikeda genotype occurred in New Zealand beginning in 2012 [20], with periparturient and lactating dairy cows and young calves exhibiting the highest morbidity and mortality [20]. T. orientalis Ikeda is now the most important cause of anemia in New Zealand cattle [7].

In the United States, T. orientalis outbreaks have been historically rare, and, until 2017, were attributed to strains closely related to the clinically benign Buffeli genotype $[1,21,22]$. In 2017, a cow-calf beef herd in Albemarle County, VA experienced an outbreak of febrile illness. Six animals died, and examination of a 7th clinically ill animal revealed anemia and lethargy. Blood from this animal was positive for T. orientalis. The Ikeda genotype was identified from the clinically affected animal and herd mates [23]. In parallel, ticks collected from a calf on the index farm were identified as H. longicornis, the first report of this tick outside of New Jersey.
H. longicornis is native to countries within East Asia, including Russia, China, Korea, and Japan [24]. As H. longicornis can survive within a diverse climactic range, and many populations are capable of parthenogenetic reproduction, it has successfully invaded and become established in many countries within the Asia-Pacific region, including Australia and New Zealand [24]. Recently, $H$. longicornis invasion of the United States has been confirmed [25]. In addition to its presence on the T. orientalis Ikeda outbreak index farm in Virginia, it has been identified in New Jersey [25], New York [26, 27], Pennsylvania [28], Maryland [28], Delaware [29], Connecticut [28], North Carolina [30], West Virginia [31], Tennessee [29], Kentucky [29], Arkansas [31], Rhode Island [29], Ohio [29], and South Carolina [29], and is believed to have been present within the eastern United States for at least 8 years, having initially been misclassified as $H$. leporispalustris [31].

In addition to $H$. longicornis, several other tick species are competent biological vectors of $T$. orientalis, including other species of Haemaphysalis ticks [3] and Rhipicephalus (Boophilus) microplus [12]. Interestingly, T. orientalis Buffeli isolated from a historical U.S. outbreak was not transmissible by Korean $H$. longicornis ticks [1, 32]. Although $H$. longicornis ticks from VA were PCRpositive for T. orientalis Ikeda [33], it is not yet known whether these ticks are the source of $T$. orientalis transmission to cattle in VA. If $H$. longicornis proves to be a competent vector of T. orientalis Ikeda in the U.S., increased tick control practices and vigilance regarding cattle health may be required to prevent disease spread and losses similar to those experienced by cattle producers in Australia and New Zealand. Thus, the objective of this study was to determine whether the strain of $H$. longicornis ticks that has spread throughout the U.S. can acquire and transmit the $T$. orientalis Ikeda-VA isolate. The results of this study will provide important information for U.S. cattle producers regarding the potential threat of pathogen transmission to cattle by the invasive Asian longhorned tick.

\section{Materials and methods \\ Cattle}

Four, 2-3 month-old Holstein-Friesian steer calves were utilized in this study. Pre-infection complete blood counts (CBCs) and serum chemistry panels were normal, and all calves tested negative on a pre-infection Anaplasma marginale/Anaplasma centrale competitive enzyme linked immunosorbent assay (cELISA) test (VMRD, Pullman, WA). PCR for the T. orientalis Ikeda major piroplasm surface protein (MPSP) (described below) performed on pre-infection peripheral blood samples from each calf was negative. The experimental 
protocol was approved by the University of Idaho Institutional Animal Care and Use Committee, Protocol number 2018-46.

One steer, \#1697, underwent splenectomy at the Washington State University Veterinary Teaching Hospital, and, to ensure full recovery, was placed on stall rest for 4 weeks before proceeding with the experiment. The splenectomy was performed under the supervision of a board-certified agricultural animal internist using standard surgical, anesthetic, and analgesic protocols. Once recovered, the calf was infected intravenously with $6 \mathrm{~mL}$ of $T$. orientalis Ikeda blood stabilate (Abermarle, VA 2018). Beginning 10 days after infection, rectal temperature, pulse, respiratory rate, lymph node size, and mucous membrane color were assessed and recorded daily, and a CBC, chemistry panel, and T. orientalis peripheral blood PCR and qPCR assays (described below) were performed weekly. Diff-Quik-stained blood smears and packed cell volume were assessed 2-3 times per week until the calf became positive by PCR, and were measured daily thereafter. Monitoring of steers and T. orientalis infection kinetics were similarly performed for tick acquisition and transmission feeds. Following tick removal and confirmation of infection, the calf was euthanized via intravenous administration of sodium pentobarbital (Fatal Plus ${ }^{\circledR}$, Vortech Pharmaceuticals, Michigan, USA).

\section{T. orientalis blood stabilate}

Whole blood from a T. orientalis Ikeda PCR-positive calf identified during the VA outbreak was collected into citrate phosphate dextrose adenine (CPDA) anticoagulant via jugular venipuncture. Following collection, blood was centrifuged at $1800 \times g$ for $10 \mathrm{~min}$, and the plasma and buffy coat removed. The remaining erythrocyte fraction was washed three times in filter-sterilized $(0.22 \mu \mathrm{m})$ Puck's saline $\mathrm{G}$ (pH 7.2). Finally, $20 \%$ polyvinylpyrrolidone-40 (Sigma, St. Louis, MO, USA) was added to the washed erythrocytes and $1.5 \mathrm{~mL}$ aliquots of the $1: 1$ blood/PVP mixture were transferred to $2 \mathrm{~mL}$ cryovials. The mixture was cryopreserved at $-80{ }^{\circ} \mathrm{C}$ for later use, and stabilates were thawed rapidly immediately prior to inoculation.

\section{H. longicornis colony maintenance}

$H$. longicornis adult and nymphal ticks were collected from the field in New Jersey by Dr. Dana Price (Rutgers University, New Jersey) and kindly provided to the USDA-ARS Animal Disease Research Unit in Pullman, WA for colony rearing. To rear all life stages, ticks were fed on Holstein-Friesian steer calves under a secure cloth or stockinette patch attached by adhesive. Fed ticks were collected and held in an incubator at $26^{\circ} \mathrm{C}$ to allow molting and tanning. Colony ticks were held for long-term storage at $15{ }^{\circ} \mathrm{C}$ and experimental ticks were maintained at $26^{\circ} \mathrm{C}$ until used for acquisition and transmission feeds. The ticks used in these experiments were reared from eggs in our facility in Moscow, ID. The colony, which is composed entirely of females and reproduces parthenogenetically, had been reared for one generation (F1) as of the date of this transmission trial.

\section{$H$. longicornis acquisition feed}

For acquisition feeding, nymphs were applied to splenectomized steer \#1697 in two batches. Prior to feeding, an $8.5^{\prime \prime} \times 8.0^{\prime \prime}$ patch divided into two equal sections was applied to the back of the calf. Batch \#1 was applied 13 days after first detection of $T$. orientalis by peripheral blood PCR (see below). Batch \#2 was applied 6 days later after visualization of piroplasms within erythrocytes on Diff-Quik-stained blood smears. For each batch, 154 nymphs were applied to one section of the cloth patch. Nymphs fed to repletion over a 4-8 day period. During this time, engorged nymphs were collected and held as described above to allow molting into adults.

\section{H. longicornis transmission feed}

Following acquisition feeding, nymphs were held for approximately 1 month to allow molting. Post-molt, adult ticks from both batches were divided evenly into three groups and allowed to feed on three, spleen-intact steers (\#1718, \#1726, and \#1727). Prior to tick application, an $8.5^{\prime \prime} \times 8.0^{\prime \prime}$ patch was applied to the right and left flanks of each calf using adhesive. For transmission feeding, 42 ticks from Batch \#1 and 28 ticks from Batch \#2 were applied to each calf in the front left and right patches, respectively.

To confirm T. orientalis acquisition and maturation, a subset of adult ticks from each batch were allowed to feed for 4 days to stimulate salivary gland development and parasite replication, and were then collected and dissected to harvest salivary glands. Salivary glands were placed into individual $1.5 \mathrm{~mL}$ microfuge tubes on ice containing Phosphate Buffered Saline (PBS; Gibco) and stored at $-20{ }^{\circ} \mathrm{C}$ until analysis by qPCR as described below.

\section{T. orientalis MPSP PCR}

Frozen samples were extracted using the QIAamp DNA mini kit (Qiagen, Hilden, Germany) following the blood or body fluids spin protocol (blood and salivary glands) or the tissue protocol (larvae) using proteinase K. EDTA anticoagulated blood $(100 \mu \mathrm{L})$ and salivary gland pairs dissected from acquisition fed adults were brought up to $200 \mu \mathrm{L}$ with PBS. Elution was performed with $50 \mu \mathrm{L}$ of pre-warmed buffer $\mathrm{AE}$ and 2 min incubation prior to centrifugation; this step was repeated for blood 
samples. Detection of the MPSP gene by conventional PCR was carried out in reactions composed of $22.5 \mu \mathrm{L}$ of AccuPrime ${ }^{\mathrm{TM}}$ Pfx SuperMix (Thermo Fisher Scientific, Waltham, MA, USA), $1.5 \mu \mathrm{L}$ of $10 \mathrm{uM}$ each MPSP primer [23] and $2 \mu \mathrm{L}$ of DNA template. Reaction conditions were as follows: 5 min at $95{ }^{\circ} \mathrm{C}$ followed by 35 cycles of $15 \mathrm{~s}$ at $95^{\circ} \mathrm{C}, 5 \mathrm{~s}$ at $57^{\circ} \mathrm{C}$ and $1 \mathrm{~min}$ at $68{ }^{\circ} \mathrm{C}$. Ten microliters of the final reaction volume was electrophoresed on a $1.25 \%$ agarose gel. Positive controls (blood stabilate) and positive samples produced fragments of the expected size of $776 \mathrm{bp}$ and negative controls showed no signs of amplification (Additional file 1: Figure S1). Positive samples (one date per animal and blood stabilate control) were verified as T. orientalis with bidirectional Sanger sequencing using PCR primers (Eurofins Genomics, Louiseville KY). Sequences were trimmed and merged for consensus to create consensus sequences (Sequencher, GeneCodes, Ann Arbor, MI, USA).

\section{T. orientalis qPCR}

Real-time PCR targeting the $T$. orientalis MPSP gene was completed on DNA extracted from blood of the four steers and from salivary glands of ticks fed on the three transmission-fed steers. PCR reaction components consisted of $\mathrm{TaqMan}^{\mathrm{TM}}$ Environmental Master Mix 2.0 (Thermo Fisher Scientific), $300 \mu \mathrm{M}$ of Forward Primer [34] (5'-GCA AAC AAG GAT TTG CAC GC-3'), $300 \mu \mathrm{M}$ of Reverse Primer (5'-TGT GAG ACT CAA TGC GCC TAG A-3'), $100 \mu \mathrm{M}$ of Probe (5'-NED-TCG ACA AGT TCT CAC CAC-MGB-NFQ-3'), and $2 \mu \mathrm{L}$ of DNA in a $20 \mu \mathrm{L}$ reaction. Serial ten-fold dilutions of plasmid DNA pASK-IBA2 (IBA Life Sciences, Goettingen, Germany) ligated with a partial $T$. orientalis Ikeda MPSP gene were included in the assay as a standard curve. qPCR was run on a 7500 Fast Real-Time PCR System (Thermo Fisher Scientific) using standard mode and the following cycling conditions: $10 \mathrm{~min}$ at $95 \mathrm{C}$ followed by 45 cycles of $15 \mathrm{~s}$ at 95C and $1 \mathrm{~min}$ at 60C (Additional file 2: table S1).

\section{Results}

\section{Infection of a splenectomized calf with $T$. orientalis Ikeda blood stabilate results in microscopically detectable parasites and anemia}

Sixty-three days following IV inoculation with $T$. orientalis Ikeda blood stabilate, calf 1697 tested positive for T. orientalis Ikeda via peripheral blood PCR (Additional file 1: Figure S1). T. orientalis merozoites were detectable in Diff-Quick stained blood smears from day 80 post-infection onward (Fig. 1a). Peripheral blood parasitemia peaked at $3.4 \% 91$ days post infection, then decreased (Fig. 2). The PCV of calf 1697 steadily declined $6 \%$, from $32 \%$ pre-infection to a nadir of $26 \%$, by 99 days post-infection (Fig. 2). T. orientalis was detected by qPCR beginning at 56 days post-infection through the end of the study at 72 days post-infection with detected copy numbers per $\mathrm{mL}$ of blood increasing steadily from $2.81 \times 10^{3}$ to $7.61 \times 10^{5}$ (Fig. 3 and Additional file 2:Table S1). As is commonly observed in $T$. orientalis infections, the calf exhibited no other clinical signs of piroplasmosis (e.g. fever, anorexia, icterus, and hemoglobinuria) throughout the course of infection.

\section{$H$. longicornis ticks fed as nymphs on a $T$. orientalis Ikeda-infected calf acquire $T$. orientalis Ikeda infection} Batch \#1 nymphs fed to repletion after approximately 4 days on T. orientalis Ikeda PCR-positive blood (Fig. 2; solid box). Batch \#2 nymphs fed to repletion after approximately 8 days, during which peripheral blood parasitemia increased from 0.1 to $1.7 \%$ (Fig. 2; dashed box). Of the total nymphs applied, 150/154 from Batch \#1 and $114 / 154$ from Batch \#2 were collected.

After molting, T. orientalis Ikeda was detected in salivary glands from a subset of adult ticks from both Batch $\# 1$ and Batch \#2 following a 4 day stimulation feed. Of the subset of stimulation-fed ticks from Batch \#1, 8/17 were positive by qPCR with an average infection rate of $47.1 \pm 12.4 \%$ and a range from $1.63 \times 10^{3}$ to $2.17 \times 10^{5}$ copies per salivary gland pair. From Batch \#2, 1/18 was positive representing an infection rate of $5.5 \%$ and $1.92 \times 10^{5}$ copies per salivary gland pair. (Fig. 4 and Additional file 2: Table S1).

\section{T. orientalis Ikeda-infected $H$. longicornis adult ticks transmit $T$. orientalis Ikeda to naïve calves}

Fourteen days after adult tick application, 3/3 spleenintact calves were positive for $T$. orientalis Ikeda via peripheral blood PCR Additional file 1: Figure S1), and merozoites were detected in the erythrocytes of all calves on day 15 post-tick application (Fig. 1b-d). T. orientalis was detected by qPCR in all three calves from 14 days post-tick application through the end of the study. Copy numbers peaked for two calves (1727 and 1726) at 28 days post-infection while copy numbers were continuing to climb steadily for the third calf (1718) at 42 days post-tick application (Fig. 5), at the end of the study. Copy numbers per $\mathrm{mL}$ of blood ranged from $1.68 \times 10^{5}$ to $1.52 \times 10^{8}$ (Additional file 2: Table S1), and for the two calves that demonstrated a peak in detected parasites, the peak was at approximately $1 \times 10^{8}$ per $\mathrm{mL}$ of blood (Fig. 5). Peripheral blood parasitemia peaked at $0.4-0.9 \%$ between 26 and 31 days post-tick application (Fig. 6), and the PCV of all calves declined $2-7 \%$ to nadirs of $28-29 \%$, followed by incremental recovery (Fig. 6). One calf (1727) developed a transient, mild fever $\left(103.5^{\circ} \mathrm{F}\right)$ on day 11 

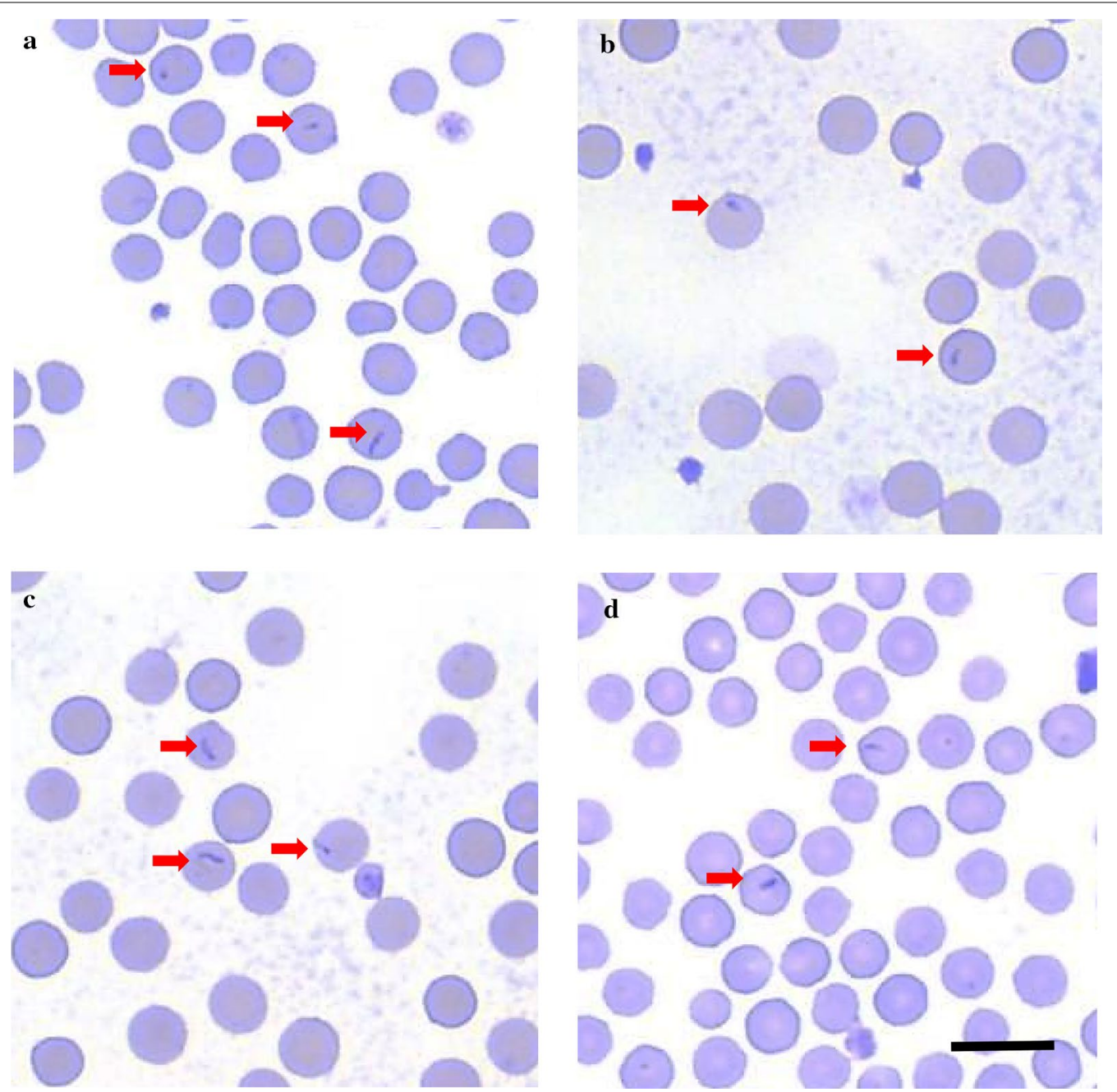

Fig. 1 Representative blood smears from calves 1697, 1718, 1726, and 1727 following T. orientalis Ikeda-infection. In each calf, few erythrocytes contain 1-2.5 $\mu \mathrm{m} \times 0.5 \mu \mathrm{m}$, tear-drop shaped, intracellular piroplasms (arrows). No other evidence of anemia, erythrocyte destruction, or cellular regeneration is present. a calf $1697, \mathbf{b}$ calf 1718 , c calf 1726 , d calf 1727 . Scale bar: $10 \mu \mathrm{m}$

post tick-application, but no other clinical signs of piroplasmosis were exhibited by the calves throughout the course of infection.

\section{Sequence anzalysis}

Consensus sequences for all sequenced amplicons were identical to each other and to that of the Ikeda strain, similar to the analysis by Oakes [23].

\section{Discussion}

These findings confirm that the U.S population of $H$. longicornis is a competent vector of $T$. orientalis Ikeda detected in beef cattle in VA, U.S. in 2017 [23]. This is the first report of T. orientalis transmission by $H$. longicornis ticks in the U.S. Our findings support the hypothesis that transmission of $T$. orientalis Ikeda within the Abermarle County, VA area is due to the abundance of $\mathrm{H}$. longicornis. The previous detection of $T$. orientalis Ikeda in $H$. longicornis, but not native ticks, from the same area in VA provides further support for this hypothesis [33].

Since the $T$. orientalis Ikeda outbreak was detected 2017, concomitant with the introduction of $H$. longicornis to the U.S., it is possible that the tick and the parasite were imported together (e.g. T. orientalis Ikedainfected $H$. longicornis ticks were imported); however, it is also possible that they were introduced separately (e.g. T. orientalis-infected cattle and uninfected H. longicornis ticks entered separately), and an outbreak occurred when the parasite and the tick arrived in the same area of VA at the same time. In general, although this has yet to be 


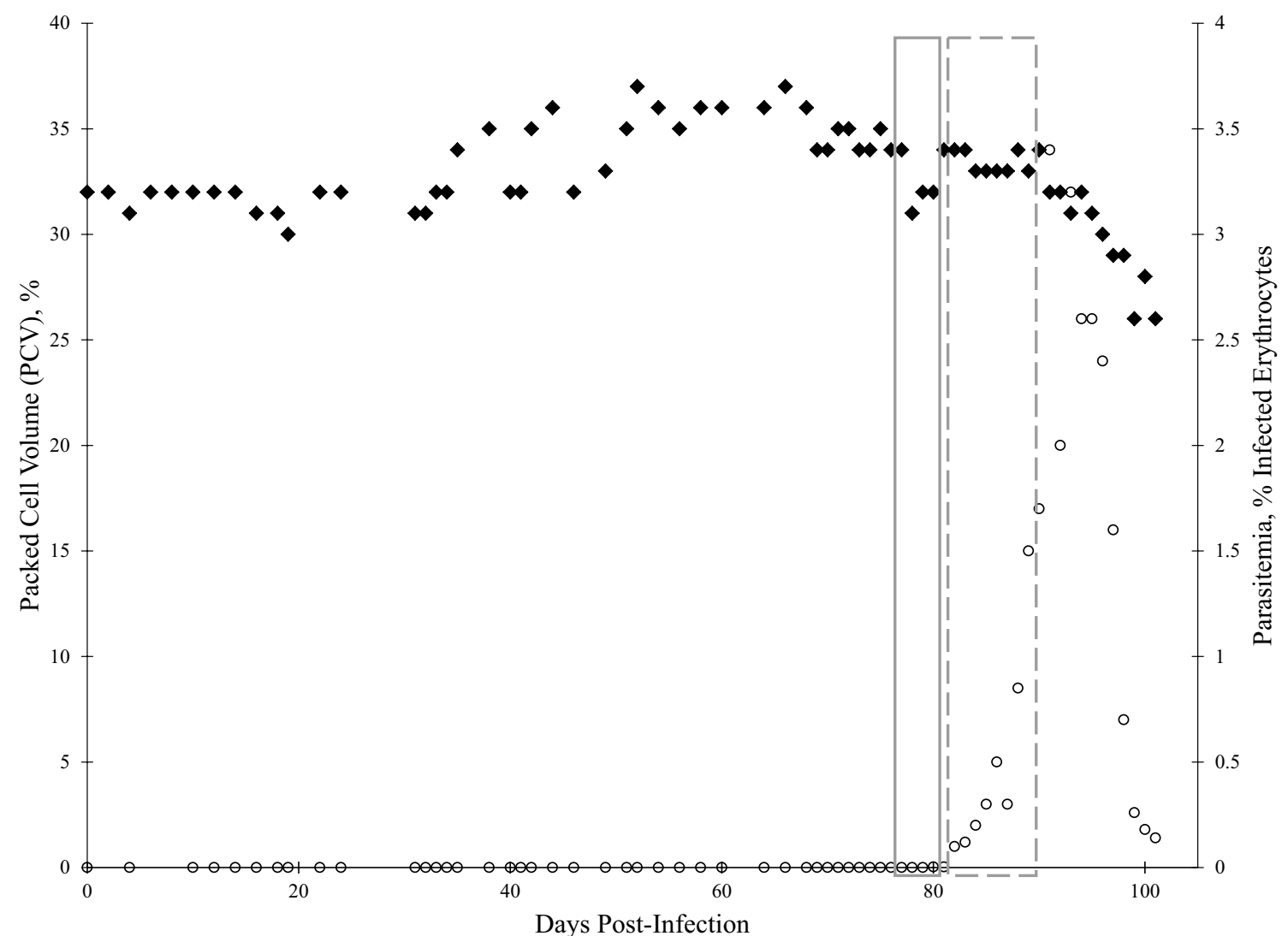

Fig. 2 Packed cell volume (PCV) and peripheral blood percent parasitemia of erythrocytes (PPE) of calf 1697 following T. orientalis Ikeda blood stabilate inoculation. PCV and Diff-quick-stained blood smears were evaluated following intravenous inoculation with T. orientalis-Ikeda-infected blood stabilate. PCV ( left y-axis) and PPE (O right y-axis) are depicted as percentages, and each value is derived from analysis of a single blood sample taken on the indicated day post-inoculation. Grey boxes indicate timeline of nymphal tick acquisition feeds. Solid box: Batch \#1, Dashed box: Batch \#2

empirically demonstrated in this strain of T. orientalis Ikeda, Theileria sp. are not transovarially transmitted. Furthermore, $H$. longicornis survives well in varied environmental conditions [24, 27, 28, 35], and exhibits a wide variety of host feeding preferences, in addition to cattle, including deer and avian species [25, 27, 28, 35]. Thus, if infected ticks were imported, an outbreak of $T$. orientalis Ikeda could occur if those infected ticks fed on cattle during their life cycle. In contrast, if the infected ticks completed their life cycle by feeding on other animals, their progeny would likely not be infected with $T$. orientalis Ikeda, and no outbreak would occur. This may explain the lack of $T$. orientalis Ikeda outbreaks in other areas of the U.S. where H. longicornis has become established [25, 27, $28,35]$.

Given the cosmopolitan nature of the tick vector, there is significant potential for extensive, rapid spread of $T$. orientalis Ikeda throughout the U.S. due to movement of infected, asymptomatic cattle. Additionally, other North American tick species competent to transmit $T$. orientalis
Ikeda are not yet known. Small-scale T. orientalis Buffeli infections have been detected in cattle herds in different areas of the U.S. since 1955 [1, 21, 22], prior to the introduction of $H$. longicornis ticks. It is thus likely some indigenous North American tick species are also capable of transmitting T. orientalis Ikeda. There is one report of $T$. orientalis acquisition and transmission by Rhipicephalus microplus ticks in India [12]. As R. microplus ticks are also native to regions of the U.S. near the southern border, and are competent vectors of Theileria equi [36-39], it is possible that they have been involved in $T$. orientalis transmission in the past, and could prove competent vectors of $T$. orientalis Ikeda. Other North American tick species, including Amblyomma mixtum, Dermacentor variabilis, and Amblyomma americanum, are competent vectors of other Theileria sp. [36, 37, 39, 40], and thus may be competent vectors of T. orientalis as well. Although T. orientalis was not detected in fieldcollected cohorts of A. americanum $(n=28)$ and $D$. variablilis $(n=10)$ ticks in VA [33], larger scale, controlled 

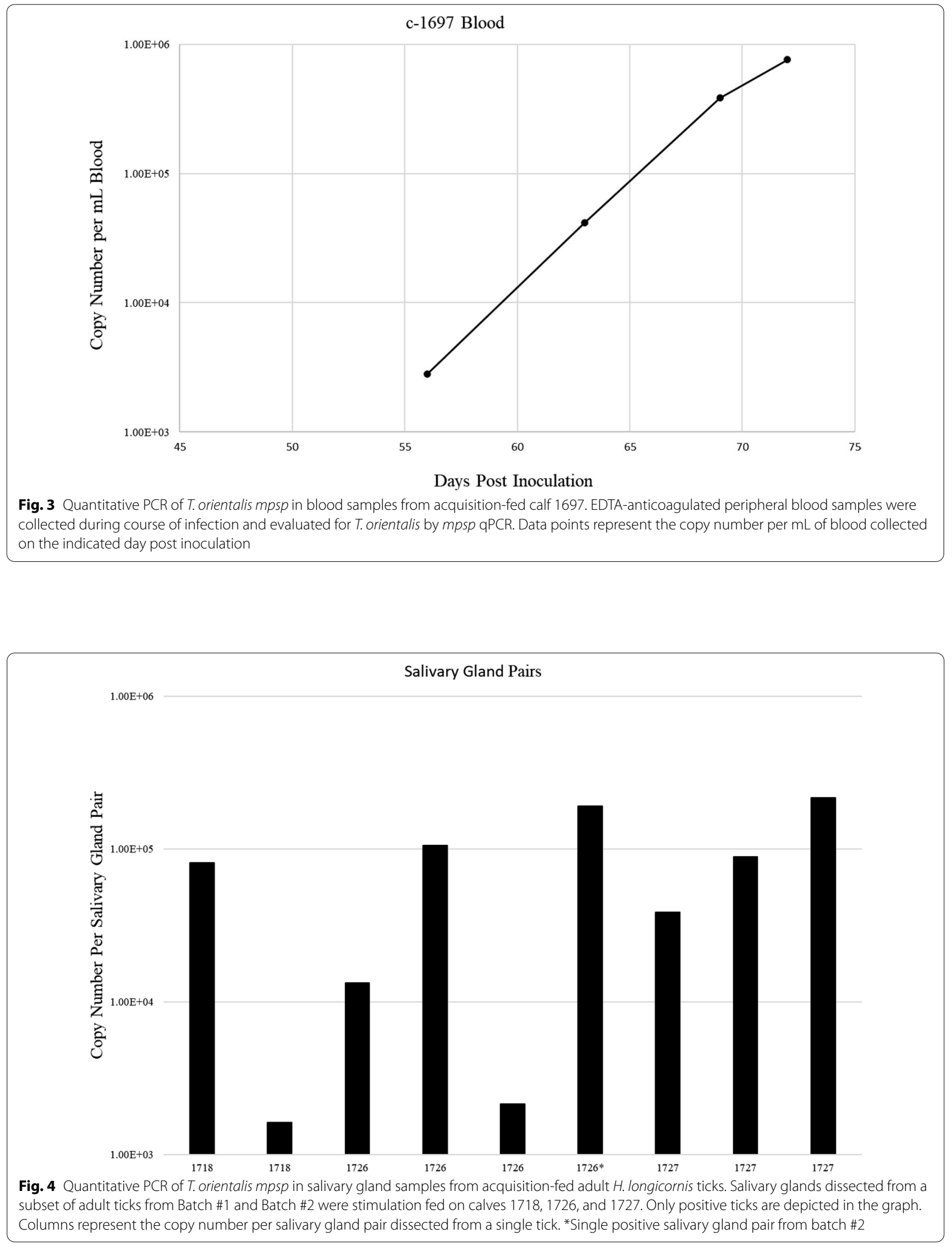

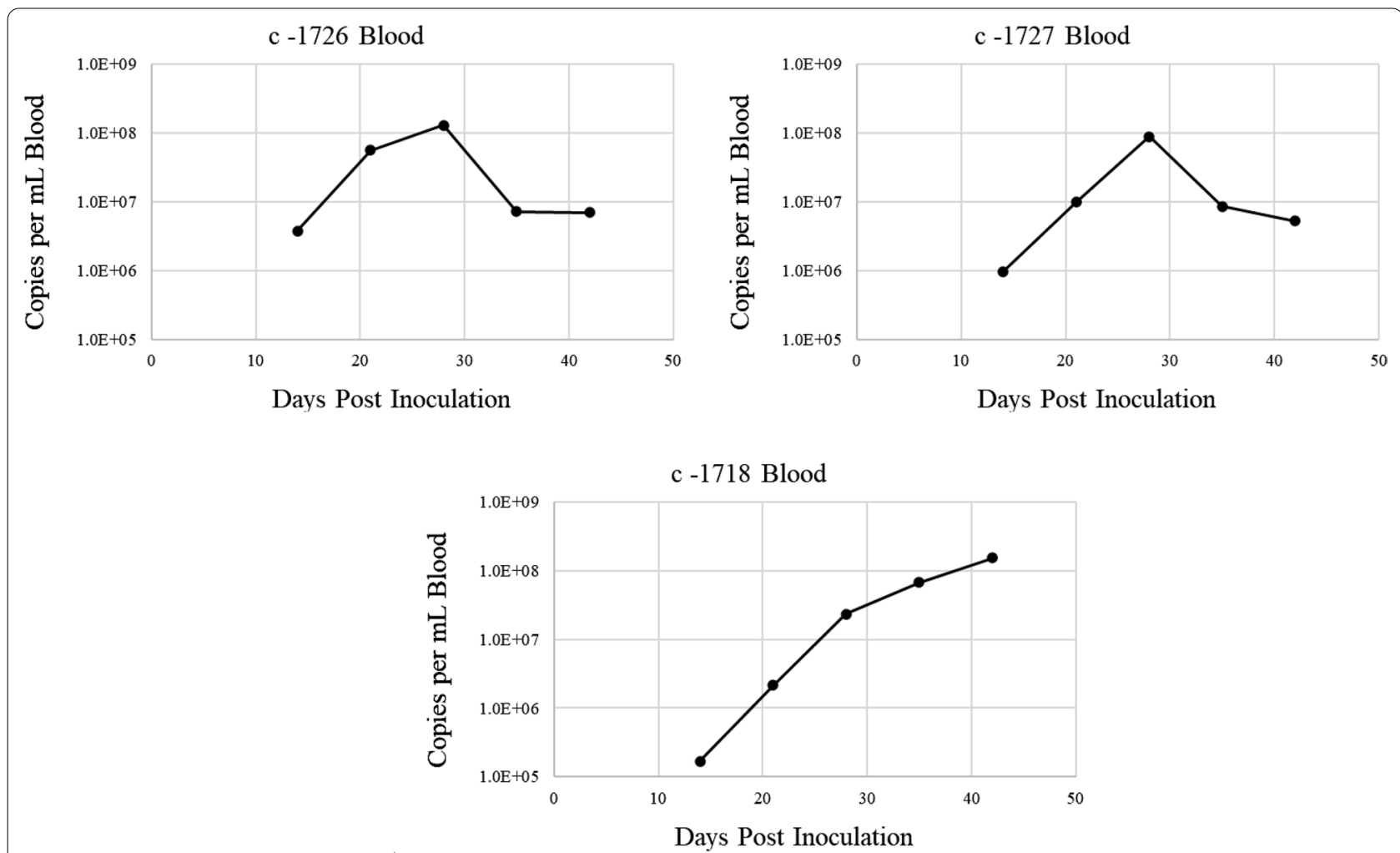

Fig. 5 Quantitative PCR analysis of T. orientalis mpsp gene fragment in blood samples from transmission-fed calves 1718, 1726, and 1727. Peripheral blood samples were evaluated as described in Fig. 3

acquisition and transmission experiments are required to definitively determine the vector competence of these tick species for T. orientalis Ikeda.

In our study, parasitemia was markedly delayed when the calf was infected via IV inoculation with blood stabilate as compared to tick feeding. There are several possible explanations for this observation. First, during tick feeding, infectious parasites are delivered to the bloodstream of the bovine within a milieu of salivary proteins that modulate the immune response and facilitate infection of cells by sporozoites [41-44]. As these modulating proteins are largely absent from infected blood stabilates, infection of host cells by parasites is less efficient. Furthermore, heterologous erythrocyte and lymphocyte antigens in the infected blood stabilate stimulate destruction of the inoculum by the recipient. Second, significant parasite amplification occurs within the tick vector, both via sexual and asexual reproduction $[45,46]$. Thus, cattle infected via tick bite likely received a higher dose of $T$. orientalis parasites. Third, when tick transmission is used for infection, parasites undergo another round of asexual reproduction within leukocytes of the host (schizogony) [3], further increasing the number of parasites that subsequently infect bovine erythrocytes, bringing animals to the threshold of PCR detection much more quickly. While small numbers of contaminating schizont-infected lymphocytes were likely present in the blood stabilates, their prevalence would have been incomparable to those observed following natural tick transmission, and most of them would have been destroyed by the immune system of the recipient calf due to expression of heterologous lymphocyte antigens. Finally, it is likely that stabilate preparation, shipment, and storage led to parasite degradation, effectively significantly decreasing the infectious parasite dose.

Disease severity due to $T$. orientalis Ikeda is variable. The three animals infected via tick feeding in this study developed mild disease in comparison to the cattle from which the isolate was derived, likely due to reduced stress, lack of exposure to other pathogens, and, most importantly, reduced parasite dose. In other virulent Theileria species, including T. parva and T. annulata, 

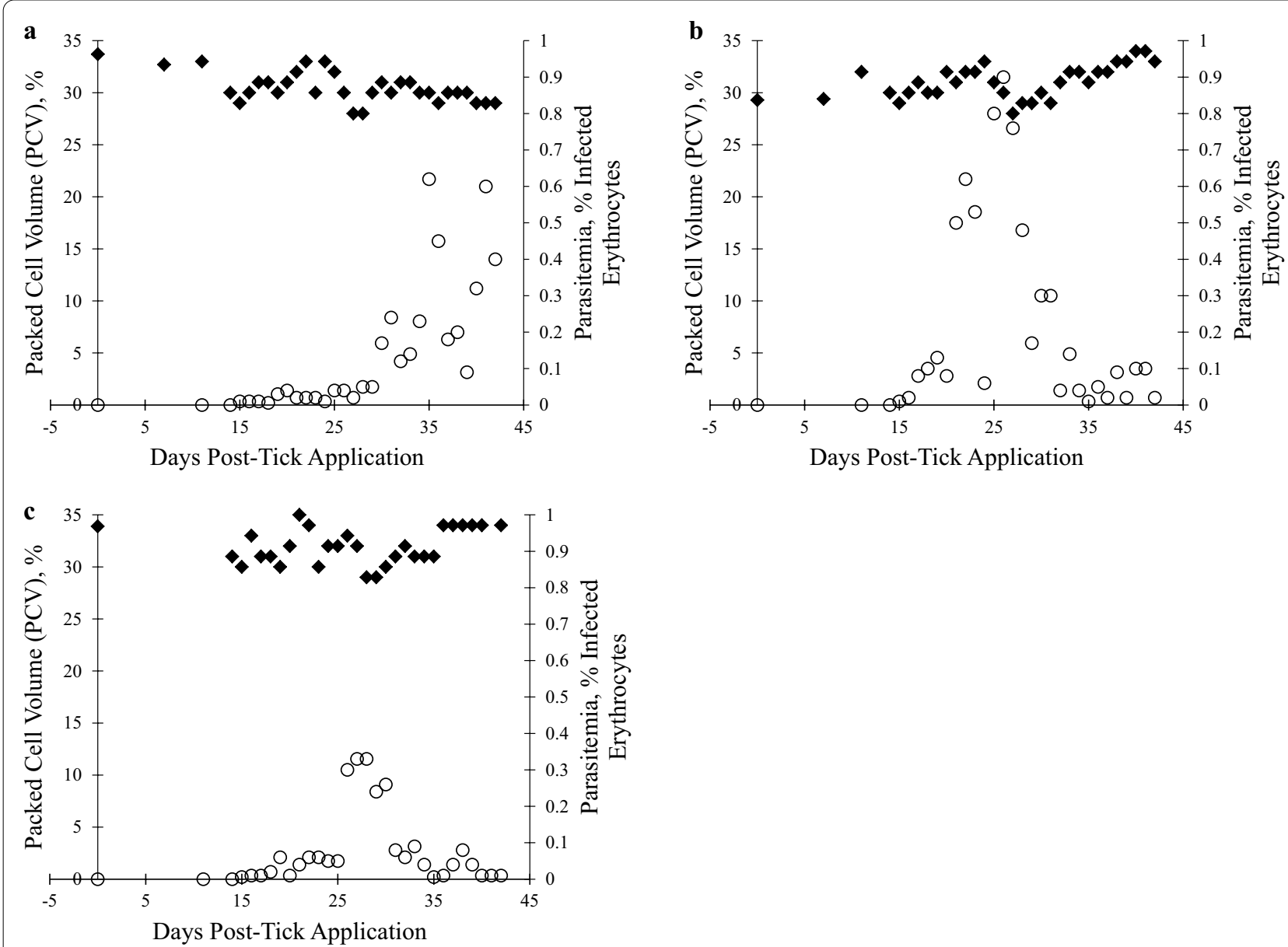

Fig. 6 Packed cell volume (PCV) and peripheral blood percent parasitemia of erythrocytes (PPE) in calves 1718, 1726, and 1727 following transmission feed by T. orientalis Ikeda-infected H. longicornis adult ticks. PCV and Diff-quick-stained blood smears were evaluated following tick attachment and feeding. a calf 1718, b calf 1726, c calf 1727. For A-C, PCV (left y-axis) and PPE (right y-axis) are depicted as percentages, and each value is derived from analysis of a single blood sample taken on the indicated day post-inoculation. $\downarrow$ PCV, O PPE

parasite dose is a critical determinant of disease lethality $[47,48]$. In $T$. orientalis-endemic areas, cattle often endure dense $H$. longicornis infestations [49], and thus may be exposed to extremely high numbers of parasites. Exposure of immunologically naïve or debilitated animals to such a high parasite dose may contribute to morbidity and mortality caused by $T$. orientalis. Cattle age and pregnancy status are also purported to play a role in $T$. orientalis disease susceptibility. For instance, in Australia and New Zealand, the majority of T. orientalis-related economic impact on the cattle industry is due to fetal loss, abortion, and neonatal calf death $[16,20,50]$.

\section{Conclusions}

In conclusion, we have demonstrated that a U.S. population of the invasive Asian longhorned tick, H. longicornis, is a competent vector of the T. orientalis Ikeda genotype isolated from a beef herd outbreak in VA, U.S. in 2017 [23]. Since the Asian longhorned tick has become established in fourteen U.S. states [25, 28, 35], and many $T$. orientalis-infected cattle exhibit only mild, intermittent clinical signs, there is significant potential for $T$. orientalis Ikeda to spread widely within the expanding North American range of $H$. longicornis in the absence of surveillance and livestock tick control measures throughout this and neighboring regions. Furthermore, the development and implementation of improved diagnostic assays for T. orientalis surveillance may be needed to reduce losses incurred by the U.S. cattle industry. Finally, future studies to determine the vector competence of other North American tick species for T. orientalis Ikeda will provide critical knowledge regarding disease transmission and susceptibility of cattle in different regions of the United States. 


\section{Supplementary Information}

The online version contains supplementary material available at https://doi. org/10.1186/s13071-021-04659-9.

Additional file 1: Figure S1. Representative agarose gel electrophoresis image of PCR amplified products of the mpsp fragment from acquisitionand transmission-fed calf blood samples. Acquisition-fed calf 1697 at 63 dpi and transmission-fed calves 1718, 1726, and 1727 at 14 dpi. POS; T. orientalis blood stabilate positive control, NEG; no template control.

Additional file 2: Table S1. T. orientalis MPSP qPCR raw data files

\section{Acknowledgements}

The authors wish to acknowledge the excellent technical support and animal care provided by Shelby Beckner, Ralph Horn, Megan Jacks, and Gavin Scoles. We also wish to thank Dr. Angela Benton (Department of Biomedical Sciences and Pathobiology, Virginia-Maryland College of Veterinary Medicine) for providing MPSP-pDNA.

\section{Authors' contributions}

$L F, K D, D H, S N, K L, M U, K M$, and $G S$ conceived and designed the experiments. $K D, D H, S M T, K L, S M T, K M$, and LF performed the experiments. $L F, K D, D H, S N$, $\mathrm{KM}, \mathrm{KL}$, and SMT analyzed the data. LF, SN, KL, SMT, KM and GS contributed with reagents/materials/analysis tools. KD, DH, KL, MU, GS, SMT and LF wrote the paper. All authors read and approved the final manuscript.

\section{Funding}

This work was supported by the Agricultural Research Service (ARS), USA, CRIS\#2090-320000-034-00D.

\section{Availability of data and materials}

All data generated or analyzed during this study are included in this published article (and its additional files).

\section{Declarations}

\section{Ethics approval and consent to participate}

The experimental protocol was approved by the University of Idaho Institutional Animal Care and Use Committee, Protocol number 2018-46.

\section{Consent for publication}

Not applicable.

\section{Competing interests}

The authors declare that they have no competing interests.

\section{Author details}

${ }^{1}$ Department of Veterinary Microbiology \& Pathology, Washington State University, Pullman, WA, USA. ${ }^{2}$ United States, Department of Agriculture, Agricultural Research Service, Animal Disease Research Unit, Pullman, WA, USA

${ }^{3}$ Department of Biomedical Sciences and Pathobiology, Virginia-Maryland College of Veterinary Medicine, Blacksburg, VA, USA. ${ }^{4}$ Present Address: United States Department of Agriculture, Agricultural Research Service, Invasive Insect Biocontrol and Behavior Laboratory, Beltsville, MD, USA.

Received: 9 October 2020 Accepted: 25 February 2021

Published online: 16 March 2021

\section{References}

1. Uilenberg G, Perie NM, Spanjer AA, Franssen FF. Theileria orientalis, a cosmopolitan blood parasite of cattle: demonstration of the schizont stage. Res Vet Sci. 1985;38(3):352-60

2. Sivakumar T, Hayashida K, Sugimoto C, Yokoyama N. Evolution and genetic diversity of Theileria. Infect Genet Evol. 2014;27:250-63. https:// doi.org/10.1016/j.meegid.2014.07.013.

3. Watts JG, Playford MC, Hickey KL. Theileria orientalis: a review. N Z Vet J. 2016;64(1):3-9. https://doi.org/10.1080/00480169.2015.1064792.
4. Kim S, Yu DH, Chae JB, Choi KS, Kim HC, Park BK, et al. Pathogenic genotype of major piroplasm surface protein associated with anemia in Theileria orientalis infection in cattle. Acta Vet Scand. 2017;59(1):51. https ://doi.org/10.1186/s13028-017-0318-8

5. Eamens GJ, Gonsalves JR, Jenkins C, Collins D, Bailey G. Theileria orientalis MPSP types in Australian cattle herds associated with outbreaks of clinical disease and their association with clinical pathology findings. Vet Parasitol. 2013;191(3-4):209-17. https://doi.org/10.1016/j.vetpar.2012.09.007.

6. Vink WD, Lawrence K, McFadden A, Bingham P. An assessment of the herd-level impact of the Theileria orientalis (Ikeda) epidemic of cattle in New Zealand, 2012-2013: a mixed methods approach. N Z Vet J. 2016;64(1):48-54. https://doi.org/10.1080/00480169.2015.1090893.

7. Lawrence KE, Forsyth SF, Vaatstra BL, McFadden A, Pulford DJ, Govindaraju $\mathrm{K}$, et al. Clinical haematology and biochemistry profiles of cattle naturally infected with Theileria orientalis Ikeda type in New Zealand. N Z Vet J. 2018:66(1):21-9. https://doi.org/10.1080/00480169.2017.1391142.

8. McFadden A, Hart M, Bueno IM, Ha HJ, Heath A, Pulford DJ. Monitoring Theileria orientalis (Ikeda)-associated bovine anaemia in affected cattle over time. Vet Parasitol. 2017;245:29-33. https://doi.org/10.1016/j.vetpa r.2017.07.035.

9. Swilks E, Fell SA, Hammer JF, Sales N, Krebs GL, Jenkins C. Transplacental transmission of Theileria orientalis occurs at a low rate in field-affected cattle: infection in utero does not appear to be a major cause of abortion. Parasit Vectors. 2017;10(1):227. https://doi.org/10.1186/s1307 1-017-2166-9.

10. Mekata H, Minamino T, Mikurino Y, Yamamoto M, Yoshida A, Nonaka N, et al. Evaluation of the natural vertical transmission of Theileria orientalis. Vet Parasitol. 2018;263:1-4. https://doi.org/10.1016/j.vetpar.2018.09.017.

11. Marendy D, Baker K, Emery D, Rolls P, Stutchbury R. Haemaphysalis longi cornis: the life-cycle on dogs and cattle, with confirmation of its vector status for Theileria orientalis in Australia. Vet Parasitol X. 2020;3:100022. https://doi.org/10.1016/j.vpoa.2019.100022.

12. Kakati P, Sarmah PC, Ray D, Bhattacharjee K, Sharma RK, Barkalita LM, et al. Emergence of oriental theileriosis in cattle and its transmission through Rhipicephalus (Boophilus) microplus in Assam. India Vet World. 2015;8(9):1099-104. https://doi.org/10.14202/vetworld.2015.1099-1104.

13. Hammer JF, Jenkins C, Bogema D, Emery D. Mechanical transfer of Theileria orientalis: possible roles of biting arthropods, colostrum and husbandry practices in disease transmission. Parasit Vectors. 2016;9:34. https://doi.org/10.1186/s13071-016-1323-x.

14. Fujisaki K, Kamio T, Kawazu S, Shimizu S, Simura K. Theileria sergenti: experimental transmission by the long-nosed cattle louse, Linognathus vituli. Ann Trop Med Parasitol. 1993;87(2):217-8.

15. Islam MK, Jabbar A, Campbell BE, Cantacessi C, Gasser RB. Bovine theileriosis-an emerging problem in south-eastern Australia? Infect Genet Evol. 2011;11(8):2095-7. https://doi.org/10.1016/j.meegid.2011.08.012.

16. Perera PK, Gasser RB, Anderson GA, Jeffers M, Bell CM, Jabbar A. Epidemiological survey following oriental theileriosis outbreaks in Victoria, Australia, on selected cattle farms. Vet Parasitol. 2013;197(3-4):509-21. https://doi.org/10.1016/j.vetpar.2013.06.023.

17. Perera PK, Gasser RB, Firestone SM, Anderson GA, Malmo J, Davis G, et al. Oriental theileriosis in dairy cows causes a significant milk production loss. Parasit Vectors. 2014:7:73. https://doi.org/10.1186/1756-3305-7-73.

18. Lawrence KE, Lawrence BL, Hickson RE, Hewitt CA, Gedye KR, Fermin $L M$, et al. Associations between Theileria orientalis Ikeda type infection and the growth rates and haematocrit of suckled beef calves in the north island of New Zealand. N Z Vet J. 2019;67(2):66-73. https://doi. org/10.1080/00480169.2018.1547227.

19. J. Lane TJ, R. Shepherd, J. Webb-Ware, G. Fordyce. Priority list of endemic diseases for the red meat industries. In: B.AHE.0010, editor. North Sydney: Meat and Livestock Australia Ltd; 2015. p. 76-81.

20. Lawrence K, McFadden A, Gias E, Pulford DJ, Pomroy WE. Epidemiology of the epidemic of bovine anaemia associated with Theileria orientalis (Ikeda) between august 2012 and march 2014. N Z Vet J. 2016;64(1):3847. https://doi.org/10.1080/00480169.2015.1090894.

21. Kakuda T, Shiki M, Kubota S, Sugimoto C, Brown WC, Kosum C, et al. Phylogeny of benign Theileria species from cattle in Thailand, China and the USA based on the major piroplasm surface protein and small subunit ribosomal RNA genes. Int J Parasitol. 1998;28(8):1261-7.

22. Stockham SL, Kjemtrup AM, Conrad PA, Schmidt DA, Scott MA, Robinson TW, et al. Theileriosis in a Missouri beef herd caused by Theileria buffeli: 
case report, herd investigation, ultrastructure, phylogenetic analysis, and experimental transmission. Vet Pathol. 2000;37(1):11-21. https://doi. org/10.1354/vp.37-1-11.

23. Oakes VJ, Yabsley MJ, Schwartz D, LeRoith T, Bissett C, Broaddus C, et al. Theileria orientalis Ikeda genotype in cattle, Virginia, USA. Emerg Infect Dis. 2019;25(9):1653-9. https://doi.org/10.3201/eid2509.190088.

24. Heath A. Biology, ecology and distribution of the tick, Haemaphysalis longicornis Neumann (Acari: Ixodidae) in New Zealand. N Z Vet J. 2016:64(1):10-20. https://doi.org/10.1080/00480169.2015.1035769.

25. Rainey T, Occi JL, Robbins RG, Egizi A. Discovery of Haemaphysalis longicornis (Ixodida: Ixodidae) parasitizing a sheep in New Jersey. United States J Med Entomol. 2018;55(3):757-9. https://doi.org/10.1093/jme/tjy006.

26. New species of tick found in New York State, Longhorned tick may pose a threat to livestock, particularly cattle. 2018. https://www.health.ny.gov/ press/releases/2018/2018-07-17_precaution_against_ticks.htm. Accessed 17 July 2018

27. Tufts DM, VanAcker MC, Fernandez MP, DeNicola A, Egizi A, DiukWasser MA. Distribution, host-seeking phenology, and host and habitat associations of Haemaphysalis longicornis ticks, Staten Island, New York, USA. Emerg Infect Dis. 2019;25(4):792-6. https://doi.org/10.3201/eid25 04.181541 .

28. Beard CB, Occi J, Bonilla DL, Egizi AM, Fonseca DM, Mertins JW, et al. Multistate infestation with the exotic disease-vector tick Haemaphysalis longicornis_United States, august 2017-september 2018. MMWR Morb Mortal Wkly Rep. 2018;67(47):1310-3. https://doi.org/10.15585/mmwr. mm6747a3.

29. National Haemaphysalis longicornis (Asian longhorned tick) situation report as of february 3, 2021. In: Service USDoAAaPHI, editor. United States Department of Agriculture Animal and Plant Health Inspection Service; 2021.

30. Longhorned tick found in Polk County, NC. NC Department of Agriculture and Consumer Services News: 1; 2018. Accessed 11 July 2018.

31. Factsheet Longhorned tick: Information for livestock and pet owners. In: USDA-APHIS, editor. USDA-APHIS; 2018. p. 2.

32. Kuttler KL, Craig TM. Isolation of a bovine Theileria. Am J Vet Res 1975;36(3):323-5.

33. Thompson AT, White S, Shaw D, Egizi A, Lahmers K, Ruder MG, et al. Theileria orientalis Ikeda in host-seeking Haemaphysalis longicornis in Virginia, U.S.A. Ticks Tick Borne Dis. 2020;11(5):101450. https://doi. org/10.1016/j.ttbdis.2020.101450.

34. Bogema DR, Deutscher AT, Fell S, Collins D, Eamens GJ, Jenkins C. Development and validation of a quantitative PCR assay using multiplexed hydrolysis probes for detection and quantification of Theileria orientalis isolates and differentiation of clinically relevant subtypes. J Clin Microbiol. 2015;53(3):941-50. https://doi.org/10.1128/JCM.03387-14.

35. Egizi A, Bulaga-Seraphin L, Alt E, Bajwa WI, Bernick J, Bickerton M, et al. First glimpse into the origin and spread of the Asian longhorned tick, Haemaphysalis longicornis, in the United States. Zoonoses Public Health. 2020. https://doi.org/10.1111/zph.12743.

36. Wise LN, Pelzel-McCluskey AM, Mealey RH, Knowles DP. Equine piroplasmosis. Vet Clin N Am Equine Pract. 2014;30(3):677-93. https://doi. org/10.1016/j.cveq.2014.08.008.

37. Wise LN, Kappmeyer LS, Mealey RH, Knowles DP. Review of equine piroplasmosis. J Vet Intern Med. 2013;27(6):1334-46. https://doi.org/10.1111/ jvim.12168.

38. Ueti MW, Palmer GH, Kappmeyer LS, Statdfield M, Scoles GA, Knowles DP. Ability of the vector tick Boophilus microplus to acquire and transmit Babesia equi following feeding on chronically infected horses with low-level parasitemia. J Clin Microbiol. 2005;43(8):3755-9. https://doi. org/10.1128/JCM.43.8.3755-3759.2005.
39. Scoles GA, Ueti MW. Vector ecology of equine piroplasmosis. Annu Rev Entomol. 2015;60:561-80. https://doi.org/10.1146/annurev-ento-01081 4-021110.

40. Scoles GA, Ueti MW. Amblyomma cajennense is an intrastadial biological vector of Theileria equi. Parasit Vectors. 2013;6(1):306. https://doi. org/10.1186/1756-3305-6-306.

41. Oyugi MO, Kinyua JK, Magiri EN, Kigoni MW, Costa EP, Githaka NW. In silico characterization and structural modeling of Dermacentor andersoni p36 immunosuppressive protein. Adv Bioinform. 2018:2018:7963401. https:// doi.org/10.1155/2018/7963401.

42. Preston SG, Majtan J, Kouremenou C, Rysnik O, Burger LF, Cabezas Cruz A et al. Novel immunomodulators from hard ticks selectively reprogramme human dendritic cell responses. PLoS Pathog. 2013;9(6):e1003450. https ://doi.org/10.1371/journal.ppat.1003450.

43. Tirloni L, Islam MS, Kim TK, Diedrich JK, Yates JR 3rd, Pinto AF, et al. Saliva from nymph and adult females of Haemaphysalis longicornis: a proteomic study. Parasit Vectors. 2015;8:338. https://doi.org/10.1186/s1307 1-015-0918-y.

44. Gwakisa P, Yoshihara K, Long To T, Gotoh H, Amano F, Momotani E. Salivary gland extract of Rhipicephalus appendiculatus ticks inhibits in vitro transcription and secretion of cytokines and production of nitric oxide by LPS-stimulated JA-4 cells. Vet Parasitol. 2001;99(1):53-61.

45. Florin-Christensen M, Schnittger L. Piroplasmids and ticks: a longlasting intimate relationship. Front Biosci. 2009;14:3064-73. https://doi. org/10.2741/3435.

46. Jalovecka M, Hajdusek O, Sojka D, Kopacek P, Malandrin L. The complexity of piroplasms life cycles. Front Cell Infect Microbiol. 2018;8:248. https:// doi.org/10.3389/fcimb.2018.00248.

47. Dolan TT, Young AS, Losos GJ, McMilian I, Minder CE, Soulsby K. Dose dependent responses of cattle to Theileria parva stabilate. Int J Parasitol. 1984;14(1):89-95.

48. Preston PM, Brown CG, Bell-Sakyi L, Richardson W, Sanderson A. Tropical theileriosis in Bos taurus and Bos taurus cross Bos indicus calves: response to infection with graded doses of sporozoites of Theileria annulata. Res Vet Sci. 1992;53(2):230-43. https://doi.org/10.1016/0034-5288(92)90115-i.

49. Hoogstraal H, Roberts FH, Kohls GM, Tipton VJ. Review of Haemaphysalis (kaiseriana) Longicornis Neumann (resurrected) of Australia, New Zealand, New Caledonia, Fiji, Japan, Korea, and northeastern China and USSR, and its parthenogenetic and bisexual populations (Ixodoidea, Ixodidae). J Parasitol. 1968:54(6):1197-213

50. Forshaw D, Alex SM, Palmer DG, Cotter J, Roberts WD, Jenkins C, et al. Theileria orientalis Ikeda genotype infection associated with anaemia, abortion and death in beef cattle in western Australia. Aust Vet J. 2020:98(7):290-7. https://doi.org/10.1111/avj.12937.

\section{Publisher's Note}

Springer Nature remains neutral with regard to jurisdictional claims in published maps and institutional affiliations.

Ready to submit your research? Choose BMC and benefit from:

- fast, convenient online submission

- thorough peer review by experienced researchers in your field

- rapid publication on acceptance

- support for research data, including large and complex data types

- gold Open Access which fosters wider collaboration and increased citations

- maximum visibility for your research: over 100M website views per year

At BMC, research is always in progress.

Learn more biomedcentral.com/submissions 